\title{
Human Wrongs in Animal Research: A Focus on Moral Injury and Reification
}

\author{
Jane Johnson \\ Research Fellow, Centre for Values Ethics and the Law in Medicine, \\ University of Sydney. Scholarly Teaching Fellow, Department of Philosophy, \\ Macquarie University, Australia \\ jane.johnson@mq.edu.au
}

\begin{abstract}
Anna Smajdor
Associate Professor of Practical Philosophy, University of Oslo, Norway anna.smajdor@ifikk.uio.no
\end{abstract}

\section{Introduction}

Most research on non-human animals (hereinafter referred to as animals) involves clear harms for the animals used, either as a direct result of research protocols or by virtue of the conditions under which the animals are kept. Arguably, however, although these harms are widely acknowledged, they have not motivated significant change to the practice of animal research. In this chapter, we focus on the damage to humans that can result from animal experimentation and how this may act as an alternative driver of change.

Humans employed in animal research, whether inside animal housing or the laboratory, confront significant stress as a result of what they routinely do as part of their job, as well as by virtue of how that work is received by "outsiders" to animal research. These workplace stressors can result in physical and psychological harms. It is well known that human patients may also be harmed as a consequence of the epistemological shortcomings of research undertaken on animals, which fails to translate to human clinical settings. Whilst we will briefly discuss these kinds of physical and psychological harms, our primary focus is the moral injury that can result from the practice of animal research. Moral injury occurs when a disregard of someone's well-being causes them harm. Typically, this is understood to encompass the kind of moral wrong that may arise from systematic injustices or from criminal or violent acts. However, moral injury is increasingly recognized as a problem for the perpetrators as well as the victims of certain acts. Moral injury, thus, also occurs when a person 
is complicit in activities that they feel are morally wrong or transgressive. Moral injury as a phenomenon, in this sense, is well established in military situations, where personnel may undertake or witness actions that would be illegal or immoral in other settings.

Using arguments derived from the work of Axel Honneth (2006), we show that animal research involves an institutionalized failure to recognize nonhuman animals that not only reifies animals but the human persons engaged in this process, diminishing the scope of their moral agency and causing moral injury. In this chapter, we begin by briefly articulating the harms to animals in research and the more conventional harms to humans that can arise as a result of animal research, before making a case for the ethical damage wrought by the failures of recognition inherent within the system of animal research. We conclude with a brief outline of our approach as a means of effecting change in animal research.

\section{Harms to Animals in Research}

It is widely acknowledged that animals frequently suffer harms when used in interventional biomedical research directed at human clinical benefit. These harms may be the direct result of research protocols or relate to the conditions in which animals are housed. Animals can experience pain and discomfort when used in toxicology testing, the development of pharmaceuticals, vaccine development, diagnostic techniques, and surgical research. The intervention itself may be the source of distress, or, if the research protocol demands it, prior infliction of an alien disease or condition on the animal may be a source of suffering. Animals used in biomedical research are routinely killed at the completion of a protocol or series of protocols. Although arguments can be made that, in itself death may not amount to a harm for non-humans, the manner in which animals are killed can be a source of concern, and there is disagreement over what constitutes humane euthanasia (Hawkins et al., 2016). Housing can be another source of harm for animals in research, since the environment in which animals are kept may negatively impact their well-being. Housing that is inexpensive, easy to handle, and clean may not provide the best environment to meet the needs of animals. Animals may be harmed by lack of access to conspecifics and adequate stimulation, the intrusion of light and noise, inappropriate cage design, and so on (National Health and Medical Research Council, 2013). Although most of these harms are well known, arguably, they have not motivated significant change in the practice of research. For the remainder of this chapter, we focus instead on harms to humans from animal research, which have received relatively little attention. 


\section{Harms to Humans - Physical}

For some time, it has been acknowledged that there are epistemological problems in translating results obtained from animal experiments into human clinical benefit. A number of reasons can be cited for this failure, including, differences in physiology and metabolism between human and non-human animals (LaFollette and Shanks, 1996); poorly conducted and inappropriately evaluated animal experiments (Perel et al., 2007; Pound et al., 2004); and animal stress due to many of the environmental factors identified above (e.g. small cage size, boredom, high levels of noise, etc.), which in turn has impact on physiology and the reliability of scientific data obtained from animals (Akhtar, Pippin and Sandusky, 2008; Baldwin, Primeau and Johnson, 2006; Burwell and Baldwin, 2006; also see in this Volume: Herrmann, 2019; Jayne and See, 2019).

Irrespective of the reasons behind failures in translation, the consequences are significant for human patients and those who work with animals. First, patients may receive treatment that is inappropriate and harmful, if such treatments have "passed" animal testing but remain dangerous to humans (Pound and Bracken, 2014). In these cases outcomes may include a heightened risk of morbidity or mortality. There are also opportunity costs associated with pursuing one form of intervention rather than another. Second, patients may not receive treatments that could be beneficial, if they have "failed" animal tests, i.e. the development of potentially fruitful interventions for humans may be cut short by unsuccessful animal trials (Pound and Bracken, 2014). In addition, research findings in animals, which have no validity for humans, can lead to the misdirection of future financial resources and research efforts (Pound and Bracken, 2014). The resources of funders, researchers, and human trial participants may be effectively wasted in pursuit of what amounts to futile lines of inquiry. These resources would be better spent on different treatments or different forms of research, such as clinical trials, epidemiological studies, and computer modelling, rather than on animal research.

Those who work in animal research are also at risk of harm. Exposure to workplace stressors is associated with a range of negative outcomes (Britt et al., 2016). People who are directly involved with animal research, whether inside animal housing or the laboratory, face challenging issues in relation to the animals in their care. These workers may witness or directly cause animals to experience discomfort, pain, and suffering as part of an experimental protocol. They may be required to infect animals with a disease, or impair their function in some way, or euthanize them at the completion of the experimental protocol. Research workers can experience a range of negative feelings and health impacts (physiological, psychological, and social) as a result of their involvement in research. During their work, some may experience guilt, 
uneasiness, or frustration, as well as grief at the death of an animal in their care (American Association for Laboratory Animal Science, 2003). The culture of secrecy that cloaks much animal research limits discussion of these challenges by workers, exacerbating the problems experienced.

For those who work as animal carers or as laboratory technicians, these difficulties may be particularly pressing. Those who are employed to look after animals, rather than carry out the research per se, have frequently chosen their careers based on a love of animals; as such, they experience the harms to animals in research as especially distressing (Birke, Arluke and Michael, 2007). Furthermore, these individuals may not have been routinized to animal research in the same way as those who have trained as researchers, so they may lack the coping mechanisms that may assist in addressing these issues (Birke, Arluke and Michael, 2007). There is limited discussion of these harms in the literature. In the remainder of this chapter, we focus on an even more neglected area of harm to humans involved in animal research, namely, moral harm.

\section{Harms to Humans - Moral}

In order to make effective use of animals in research, those who work with them must, to some extent, treat them as objects: objects of scientific interest. In order to do this, the subjectivity of the animal is disregarded or denied. Its value comes not from what is intrinsic to it but from what others deem to be useful. The animal is controlled, monitored, manipulated, and measured in ways that, as we have suggested, often cause harm. This is not the same as, for example, deliberate cruelty, sadism, or vindictiveness. The intention is not usually to cause suffering but to achieve some other goal, for which the animal's suffering is a necessary prerequisite or side effect. The animal is merely a means to a scientific end, and those who are involved in the research must ensure that they are able to view animals in this narrow way and treat them accordingly.

The treatment of human beings as objects or as mere means to scientific ends is uncontroversially regarded as morally problematic. The validity of the animal model aside, whether it is morally wrong to use animals in this way depends partly on what moral theory one subscribes to. Most of those who find it acceptable to use animals for research base their reasoning on the idea that animals have a different moral status from human beings. Accordingly, much of the debate about animal rights has revolved around the question of what capacities are necessary or sufficient for full moral status, and whether animals have these capacities (Bastian et al., 2012; DeGrazia, 1996; Singer, 2013). However, we suggest that there are moral problems associated with the use of 
animals in research, regardless of their moral status. This is because, in order to make use of them, we have to adopt a particular stance towards them that requires a subjugation or diminution of our own moral agency. We can choose to treat animals as subjects or as objects for our use. When we choose the latter option, we reify them. Reification is a term with a complex political and philosophical history. For the purposes of our discussion, we build primarily on Honneth's use of the term (2006).

What Is Reification and How Does It Relate to Other Moral Concepts?

Reification is a disposition or a mode of relating to others that can be a product of systems and institutions that compel people to behave in certain ways, to treat others as mere things. It is, as Axel Honneth (2006) puts it, a social pathology (p. 92). The concept of reification has some resonance with Kant's formula of humanity. Kant insists that we should never treat other human beings as mere means to our own ends, but always as ends in themselves. Reification also has some resonance with the concepts of commodification, objectification and inattention. Elisabeth Anderson (1990), for example, discusses the commodification of women's labor in surrogacy. Commodification is bad, she says, because it is mistaken. We fail to value the commodified person and this is an error. The woman is inappropriately used - treated as a thing - rather than respected. Anderson's view suggests that there are fixed moral categories, and that we sometimes make mistakes in determining how to categorize others. This implies that commodification is not intrinsically wrong, only when we commodify the wrong entity. This is what occurs in surrogacy, according to Anderson, whereas she may not think it wrong to commodify animals used in research. We employ Honneth's (2006) approach to argue a broader and more agnostic view. Given that we are sometimes uncertain of how to respond to others, and we know that we are fallible and self-interested, to cultivate a commodifying disposition may be intrinsically, morally problematic.

Reification has similarities with what Kathie Jenni calls, vices of inattention (2003). Jenni argues that it is through inattention that people who are horrified when they know about factory farming, nevertheless, eat meat and try to avoid thinking about the horrors involved in its production. Similar claims may be made with regard to our reluctance to think about or discuss animal research. Again, this suggests a specifically epistemological kind of problem: we lack knowledge because we choose to look away instead of properly observing. 
In Anderson's (1990) account too, we make an epistemological mistake in miscategorizing certain others. However, focusing on accidents, lack of attention, and epistemological mistakes does not adequately capture the very deliberate aspects of what occurs in animal research. It is for this reason that we find reification a more compelling descriptor of the situation.

What is involved in animal research is not accidental. Indeed, reification goes hand in hand with a very specific form of attention, certainly in science. It is a reifying attention that denies not only the non-thing-ness of the object of research, but also the moral agency of the researcher, since the moral relationship between researcher and research object is fixed by institutional and external factors. The researcher cannot choose to relate to the animal as a non-thing, at least not without sacrificing the scientific mantle.

In developing his understanding of reification, Honneth (2006) discusses Lukács' view of a world where caring has been subverted and replaced with a pathological tendency towards reification (Lukács, 1971). Honneth rejects the concept of care as the counterpart of reification, in favor of the term recognition. The phenomenon of reification and the means of addressing it are central to our purposes, as we examine the ways in which animals are used in research. Reification, according to Axel Honneth, is a deadening tendency that distorts our ability to relate to the world around us. "[T]he subject is no longer empathetically engaged in interaction with its surroundings but is instead placed in the perspective of a neutral observer, psychically and existentially untouched by its surroundings" (2006, pp. 98-99).

Reification is not inherent in specific actions but in a conjunction of the action and disposition/intention. For this reason, there is no single means by which we can point at a class of actions and say they are always wrong. However, as we have suggested, it seems fairly clear that the scientific gaze is likely to be a reifying one, even before any action has been taken. Indeed, doing nothing can be compatible with reifying, if the reason for doing nothing is that one regards the entity that is being ignored as a mere thing; for example, if one fails to rescue an animal from a burning building.

It should be clear from our discussion so far that reification is deeply risky for those who are reified. Whether human or animal, their interests, suffering, and subjectivity are likely to go unnoticed or to be systematically devalued. But the moral problems stemming from reification are not limited, specifically, to the harm that it may cause to those who are reified. This is of particular importance to our analysis of the human harms engendered through animal research. Many people believe that provided certain standards of welfare are met, and research protocols are subjected to ethical review, animal research is not in itself unethical. However, animals remain research objects, and their life 
and well-being are precarious, since at the discretion of the researcher, they may be harmed or euthanized. Indeed, one of the clearest indications that research animals are reified is the fact that, once their value to an experiment is over, they are generally terminated. Animals will usually fight to preserve their existence; but the intrinsic value of an animal's life to the animal itself is not sufficient, in the research context, to allow it to live. We suggest that animal research will remain morally problematic even if issues of welfare continue to be improved, precisely because the harm suffered by research animals is only a subset of the problem. As long as research involves the reification of other animals, it will cause moral difficulties for those engaged in this research. It is here that the concept of reification is particularly significant in helping us move away from limited questions concerning the capacities or moral status of animals. From Honneth's perspective, this is irrelevant in at least one important sense. "The things we encounter in our everyday dealings with the world must also be regarded as entities to which we relate in an inappropriate way when we apprehend them merely neutrally and according to external criteria" (2006, p. 132).

We have outlined the ways in which animal research involves the reification of animals. However, a key part of our argument is that this, in turn, impacts the people responsible for working with such animals. Reification, aside from anything else, is a diminution, denial, or abrogation of moral agency. This can work in two ways. First, the reifier denies that the entity in question is anything other than a thing. Second, the very process of reification reflects back on the moral agent. The person, who has the capacity to be a moral agent, comes to feel and act as though this were not the case through reifying both their own moral agency and the entities that they encounter.

This kind of situation may lead individuals into difficulties regarding whether to continue to do work they find troubling. For example, some of those who work in the animal house and as laboratory technicians construe themselves as a type of intermediary between scientific researchers and animals, advocating and protecting the latter (Birke, Arluke and Michael, 2007). This sets up a kind of cognitive dissonance, which can be exacerbated by pressure from within the organization. For example, informal advice to management from the American Association for Laboratory Animal Science (2003) suggests supervisors remind workers that "if they cannot perform an assigned task, someone else will be required to do so" (p. 3). This means that individuals who care for the 
animals they work with, and who have built a rapport with them, can become caught in a cycle whereby they feel personally obliged and institutionally pressured to persevere with this work in spite of the personal cost.

Staff who do care about the animals they work with are themselves being reified by systemic and institutional pressures. The moral agency that enables them to relate to the animal, to have a view as to how and whether something should be done, may be stultified over time. Habermas also identifies this problem - that of our capacity to reify ourselves - calling it the "self-instrumentalization of the species" (2014). While Habermas was not concerned with animal research, he, nevertheless, offers a clear illustration of the phenomenon he was concerned with. In seeking to instrumentalize other species, we simultaneously do the same to ourselves.

\section{$7 \quad$ Loss of Moral Agency Leads to Moral Injury}

The use of animals in research requires a narrowing of the social sphere, to exclude some entities or to limit the ways in which the interests of these entities can serve to restrict our freedoms to act on them. In this way, moral agency is constrained. In addition, the nature of scientific work often means that people carry out procedures that have been defined and required by others, so that moral implications, in relation to animals, may be doubly removed from the individual's own sense of moral autonomy or agency. There are some parallels here with the known problem of desensitization: those who cannot successfully desensitize themselves to animal suffering are unlikely to thrive in jobs that require animal research. Therefore there is an inbuilt incentive for scientists and researchers to seek to desensitize themselves actively, by reframing their moral relationship with the animals (Capaldo, 2004).

We hypothesize that habitually narrowing the scope of moral concern is a source of moral injury to those who do it. Institutions and workplaces often require this kind of narrowing. For example, to promote efficiencies, effort is expended on an ever-smaller circle of those who matter. Thus, workers may find themselves told to ignore the mold on the tomatoes in the burgers, or to give parents misleading feedback on their children's reports, or to prioritize the management's targets above any other concern they have relating to the patient (Smajdor, 2013). There are many areas of modern life, maybe even most workplaces, where the demands of efficiency are such that reification seems inescapable. To this extent, the situation of those involved in animal research may not be significantly different from those working in factories or universities or engaged in other sorts of scientific or laboratory work. However, there 
is an important additional factor that feeds into the mix here. There are some people whose occupations also involve intensely, ethically-charged decisions or practices. These include those working as military personnel, medical professionals, and - we would argue - those involved in animal research.

The damaging effects of breaking strong moral taboos have previously been discussed in the context of military and medical personnel. For example, both medics and military personnel are more likely to witness, bring about, or be involved in the death of other human beings. Both are required to perform actions that cause harm to other individuals. Both may have to overcome feelings of repugnance for what they do and to attempt to distance themselves from normal human responses (Howe, Smajdor and Stöckl, 2012; Smajdor, Salter and Stöckl, 2010). For these reasons and others, medical and military personnel are at risk of moral injury (Litz et al., 2009; Steenkamp et al., 2011), and a variety of strategies have been developed in order to encourage institutions and individuals to identify these risks and deal with them. It may not be immediately obvious that one can draw parallels between animal researchers and soldiers or medics; but, in fact, proximity with death and witnessing or causing trauma is likely to be part of all of these worlds, as is the need to function within highly complex and hierarchical systems. These systems impose their own moral demands and codes, which frequently conflict with the norms and expectations of society. In these circumstances, a combination of strong social taboo attached to the activity, reification, loss of agency, and the ethical complexity of the role lends itself to a far higher risk of moral injury.

It is routinely accepted in modern societies that killing and harming animals is prima facie wrong. Indeed, to participate in activities such as these is usually against the law and/or regarded as immoral, unless carried out by designated people. Even, or especially, in developed Western societies, whose farming practices and research activities require that animals are used, killed, or harmed, members of the public are not commonly involved in these practices. What this means is that, just as doctors or members of the military are engaged in breaking taboos, so too are people whose roles involve using or harming animals. Animal researchers must contain their "normal" feelings, to some extent, and refrain from "normal" moral and social judgements just as soldiers do. Shifting between different moral contexts can, in itself, be a risk for moral injury. The switch from war to civilian life is well recognized as a source of stress, and this shift is one that animal researchers may undergo on a lesser scale every day. In some senses, animal research is even more morally taboo than military service. Soldiers can be, and often are, viewed as heroes. In the contemporary environment, it is hard to envisage the same possibility for animal researchers (Birke, Arluke and Michael, 2007, however, note that some 
pro-research campaigns seek to cast researchers as heroes for saving patients, especially children). Furthermore, soldiers are often regarded as paradigmatic examples of powerful, attractive men. It is not generally thought shameful to be a soldier. Nor would one expect to have to keep this secret. Yet, away from their colleagues, some animal researchers might feel shame, social stigma, and exclusion based on what they do. As a result, they may feel impelled to keep the nature of their work secret. Again, this taboo bears particularly on laboratory technicians as opposed to researchers, since for the former group working with animals in research constitutes the entirety of their role (Birke et al., 2007).

Aside from the elements of cognitive dissonance or shame attached to animal research, which is perhaps a result of its problematic moral status in society, moral injury may arise in this context from a more direct and personal feeling of being involved in wrongdoing. Again, this has parallels with military situations. Moral injury can result from witnessing or being complicit in acts that one feels to be wrong. One does not have to be a perpetrator of the act in question in order to be damaged by it. A sense of helplessness, or perception of being disempowered by the structures and systems within which one works, can lead to situations where one's moral agency comes under threat. Over time, this leads to a gradual hardening, or dissociation, as individuals try to protect themselves from the sense of wrongdoing and become passive and disempowered. If this dissociation is effective, a person may cease to feel distress but may, nevertheless, continue to be damaged physiologically and psychologically (Kammeyer-Mueller, Simon and Rich, 2010; Litz et al., 2009).

Several strategies and remedies could be devised to limit or ameliorate reification and its associated moral harms in the context of animal experimentation. For example, universities, hospitals, and other institutions where animal research takes place could better acknowledge the kind of stresses and pressures placed on their workers and implement policies to support resilience, perhaps akin to those adopted in the military setting. Although this may help workers cope with the issues they confront (which is not insignificant), it does not seem to get to the heart of the problem, namely, that biomedical research requires the reification of animals and, in turn, the humans who work with them. Another strategy may be to radically transform the practice of research in such a way that the harms to animals are minimized and their intrinsic value and subjectivity acknowledged. This could be facilitated by adopting the animals-as-patients model argued for elsewhere (Johnson and Degeling, 2012). 
Animal patients shift the balance of harms to benefits for animals in research and address some of the epistemological worries about the failure of animal research to translate into human clinical benefit. A move towards regarding animals as patients could represent one point along the way to a paradigm shift in animal research. This, if successful, would radically alter the relationship between researchers and participants. It would no longer be necessary for researchers to distance themselves from the animals' suffering, and, as with research involving humans, the moral value of the research participant would be an inbuilt aspect of the process.

It seems to us that, as with other major social shifts on complex issues, there will not be a single knock down argument or historical, political, or economic circumstance that will provoke change in animal research. Rather, change will occur when a number of arguments and factors come together that all support a new direction. We hope to have shown that there is a new argument that can be mounted against animal research, one that is grounded in an acknowledgement of the moral harms to humans that can result from involvement in animal experimentation. Contributing an argument that appeals to human self-interest and does not depend on problematic attempts to establish the moral status of animals or on reducing animals to their welfare, is, we hope, promising and able to further gird a move away from the current, deeply problematic, practice of animal research.

\section{References}

Akhtar, A.Z., J.J. Pippin and C.B. Sandusky (2008). Animal Models in Spinal Cord Injury: a Review. Reviews in the Neurosciences 19, pp. 47-6o.

American Association for Laboratory Animal Science (2003). Cost of caring: Human emotions in the care of laboratory animals. Memphis, TN: American Association for Laboratory Animal Science.

Anderson, E.S. (1990). Is Women's Labor a Commodity? Philosophy \& Public Affairs, 1, pp. 71-92.

Baldwin, A.L., R.L. Primeau and W.E. Johnson (2006). Effect of Noise on the Morphology of the Intestinal Mucosa in Laboratory Rats. Journal of the American Association for Laboratory Animal Science, 45, pp. 74-82.

Bastian, B., S. Loughnan, N. Haslam and H.R. Radke (2012). Don't Mind Meat? The Denial of Mind to Animals Used for Human Consumption. Personality and Social Psychology Bulletin, 38(2), pp. 247-256.

Birke, L., A. Arluke and M. Michael (2007). The sacrifice: How scientific experiments transform animals and people. West Lafayette, In: Purdue University Press. 
Britt, T.W., M. Crane, S.E. Hodson and A.B. Adler (2016). Effective and Ineffective Coping Strategies in a Low-autonomy Work Environment. Journal of Occupational Health Psychology, 21(2), pp. 154-168.

Burwell, A.K. and A.L. Baldwin (2006). Do Audible and Ultrasonic Sounds of Intensities Common in Animal Facilities Affect the Autonomic Nervous System of Rodents? Journal of Applied Animal Welfare Science, 9, pp. 179-200.

Capaldo, T. (2004) The Psychological Effects on Students of Using Animals in Ways That They See as Ethically, Morally, or Religiously Wrong. Alternatives to Laboratory Animals, 1(32), pp. 525-532.

DeGrazia, D. (1996). Taking animals seriously: Mental life and moral status. Chicago, IL: Cambridge University Press.

Habermas, J. (2014). The future of human nature. Cambridge, UK: Polity Press.

Hawkins, P., M.J. Prescott, L. Carbone, N. Dennison, C. Johnson, I.J. Makowska, N. Marquardt, G. Readman, D.M. Weary and H.D.R. Golledge (2016). A Good Death? Report of the Second Newcastle Meeting on Laboratory Animal Euthanasia. Animals, 6, p. 50.

Herrmann, K. (2019). Refinement on the way towards replacement: Are we doing what we can? In: K. Herrmann and K. Jayne, eds., Animal Experimentation: Working Towards a Paradigm Change, Vol. 22. Leiden: Brill Human Animal Studies Series, pp. $3^{-64 .}$

Honneth, A. (2006). Reification: A Recognition Theoretical View. The Tanner Lectures on Human Values. University of California, Berkeley. [online] Available at: https:// tannerlectures.utah.edu/_documents/a-to-z/h/Honneth_2006.pdf [Accessed 12/02/2018].

Howe, A., A. Smajdor and A. Stöckl (2012). Towards an Understanding of Resilience and Its Relevance to Medical Training. Medical Education, 46(4), pp. 349-356.

Jayne, K. and A. See (2019). Behavioral Research on Captive Animals: Scientific and Ethical Concerns. In: K. Herrmann and K. Jayne, eds., Animal Experimentation: Working Towards a Paradigm Change, Vol. 22. Leiden: Brill Human Animal Studies Series, pp. 517-547.

Jenni, K. (2003). Vices of Inattention. Journal of Applied Philosophy, 2o(3), pp. 279-295. Johnson, J. and C. Degeling (2012). Animals-as-Patients: Improving the Practice of Animal Experimentation. Between the Species, 15(1), pp. 43-58.

Kammeyer-Mueller, J.D., L.S. Simon and B.L. Rich (2010). The Psychic Cost of Doing Wrong: Ethical Conflict, Divestiture Socialization, and Emotional Exhaustion. Journal of Management, 38(3), pp. 784-808.

LaFollette, H. and N. Shanks (1996) Brute science. London: Routledge.

Litz, B.T., N. Stein, E. Delaney, L. Lebowitz, W.P. Nash, C. Silva and S. Maguen (2009). Moral Injury and Moral Repair in War Veterans: A Preliminary Model and Intervention Strategy. Clinical Psychology Review, 29(8), pp. 695-706. 
Lukács, G. (1971). Reification and the consciousness of the proletariat. In G. Lukács. History and Class Consciousness: Studies in Marxist Dialectics. Cambridge, MA: The MIT Press.

National Health and Medical Research Council. (2013). Australian code for the care and use of animals for scientific purposes, 8th ed. Canberra: National Health and Medical Research Council.

Perel, P., I. Roberts, E. Sena, P. Wheble, C. Briscoe, P. Sandercock, M. Macleod, L.E. Mignini, P. Jayaram and K.S. Khan (2007). Comparison of Treatment Effects Between Animal Experiments and Clinical Trials: Systematic Review. British Medical Journal, 334, pp. 197-206.

Pound, P. and M. Bracken (2014). Is Animal Research Sufficiently Evidence Based to be a Cornerstone of Biomedical Research? British Medical Journal, 348, g3387.

Pound, P., S. Ebrahim, P. Sandercock, M.B. Bracken and I. Roberts (2004). Where Is the Evidence That Animal Research Benefits Humans? British Medical Journal, 328, pp. 514-517.

Singer, P. (2013). In defense of animals: The second wave. Oxford, UK: Blackwell Publishing.

Smajdor, A. (2013). Reification and Compassion in Medicine: A Tale of Two Systems. Clinical Ethics, 8, pp. 111-118.

Smajdor, A., C. Salter and A. Stöckl (2010). The Limits of Empathy: Problems in Medical Education and Practice. Journal of Medical Ethics, 37, pp. 380-383.

Steenkamp, M.M., B.T. Litz, M.J. Gray, L. Lebowitz, W. Nash, L. Conoscenti and A. Lang (2011). A Brief Exposure-based Intervention for Service Members with PTSD. Cognitive and Behavioral Practice, 18(1), pp. 98-107. 